\title{
Tibial First Technique Using Predictive Balancing Improves Joint Balance Compared To Measured Resection
}

\author{
Edgar A. Wakelin ${ }^{1}$, Sami Shalhoub ${ }^{1}$, Jan A. Koenig ${ }^{2}$, Christopher Plaskos ${ }^{1}$ \\ ${ }^{1}$ Corin USA - Raynham, USA \\ ${ }^{2}$ Winthrop University Hospital - Rockville Centre, USA \\ edgar.wakelindcoringroup.com
}

\begin{abstract}
A well-balanced knee is a critical goal for total knee arthroplasty (TKA) across all surgical technique. Some controversy exists however about the method in which various techniques achieve soft tissue balance. Technology for balancing gaps in the tibia first approach has progressed from manual tensioners and laminar spreaders to digital tensioning tools integrated with robot-assisted navigation platforms, however there has been no comparison between femur first measured resection and tibia first techniques with this new technology. Our objective was to prospectively investigate joint balance accuracy and precision between a femur first measured resection and tibia first gap balancing technique utilizing a digital gap balancing tool.

A consecutive series cohort undergoing TKA were investigated. The cohort was divided in to 4 groups: 1) Femur First Blinded to gap data, 2) Femur First Non-Blinded to gap data, 3) Tibia first Blinded to gap data, 4) Tibia first Non-Blinded to gap data. Implanted medio-lateral (ML) gap difference was used to calculate joint balance.

94 patients were enrolled. No significant difference was found between the average ML gaps, however, the frequency of outlier balance throughout flexion, was significantly reduced in Group 4. Significant differences in the variance of the post-operative gap balance were also found, in which Group 4, reported reduced variance compared to group 3 and 2 throughout flexion and compared to group 1 at $90^{\circ}$.

Ideal joint balance may remain a controversial topic, nonetheless a tibia first approach with digital balancing allows surgeons to accurately achieve a desired joint balance target.
\end{abstract}

\section{Introduction}

A well-balanced knee is a critical goal for total knee arthroplasty (TKA) across all surgical techniques [1]. Some controversy exists however about the method in which various techniques achieve 
Tibial First Technique Using Predictive Balancing Improves Joint Balance Compared... Wakelin et al.

soft tissue balance[2]. Femur first measured resection targets mechanical alignment followed by soft tissue release, whereas tibia first gap balancing allows the femoral component alignment to vary on a patient specific basis to individually balance the flexion and extension gaps. Technology for balancing gaps in the tibia first approach has progressed from manual tensioners and laminar spreaders to digital tensioning tools integrated with robot-assisted navigation platforms [3], however there has been no comparison between femur first and tibia first techniques with this new technology.

Our objective was to prospectively investigate joint balance accuracy and precision between a femur first measured resection and tibia first gap balancing technique utilizing a digital gap balancing tool.

\section{Methods}

A consecutive series cohort undergoing robotic-assisted TKA were included in this study. All surgeries were performed using Apex Ultra-congruent components and captured joint balance data during trialing with a digital gap balancing device (Corin BalanceBot). The cohort was divided in to 4 groups: 1) Femur First in which the surgeon balances the knee blinded to digital gap data, 2) Femur First in which the surgeon balances the knee using digital gap data after all resections, 3) Tibia First using static spacer blocks to assess and balance gaps at $0^{\circ}$ and $90^{\circ}$ prior to femoral planning, 4) Tibia first using the digital tensioning tool to assess gaps throughout the range of motion wherein mediolateral gaps were planned to be equal within $2 \mathrm{~mm}$ before the femoral resection. 94 patients were enrolled in this study, Group 1: 22, Group 2: 37, Group 3: 21, Group 4: 14.

Final gap measurements were obtained in all groups throughout flexion. Medio-lateral (ML) gap difference was used to calculate joint balance at $10^{\circ}, 40^{\circ}$ and $90^{\circ}$. A gap difference of $>2 \mathrm{~mm}$ was considered an outlier. Wilcox rank sum tests were used to calculate differences in average gap balance, chi-square tests for outlier frequency and f-tests for differences in variance between the 4 groups.

\section{Results}

No significant difference was found between the average ML gaps in extension (1: $1.6 \pm 1.3 \mathrm{~mm}$, 2: $2.1 \pm 1.6 \mathrm{~mm}, 3: 1.9 \pm 1.5 \mathrm{~mm}, 4: 1.0 \pm 0.7)$, midflexion $(1: 1.5 \pm 0.9 \mathrm{~mm}, 2: 1.7 \pm 0.9 \mathrm{~mm}, 3: 1.7 \pm$ $1.0 \mathrm{~mm}, 4: 0.8 \pm 0.6)$ or flexion $(1: 2.0 \pm 1.7 \mathrm{~mm}, 2: 1.5 \pm 1.6 \mathrm{~mm}, 3: 2.0 \pm 1.7 \mathrm{~mm}, 4: 1.0 \pm 0.6)$, see figure $1 \mathrm{~A}$. The frequency of outlier balance throughout flexion however, is significantly reduced in Group 4 (10\%) compared to Group 1 (30\%), Group 2 (35\%) and Group $3(35 \%), p=0.026$.

Significant differences in the variance of the post-operative gap balance was found, figure $1 \mathrm{~B}$, in which Group 4, reported reduced variance compared to group 3 and 2 throughout flexion $\left(10^{\circ}\right.$ : Group 2: $1.5 \mathrm{~mm}$, Group 3: $1.5 \mathrm{~mm}$, Group 4: $1.1 \mathrm{~mm}, \mathrm{p}=0.022$ and 0.039 respectively, $40^{\circ}$ : Group 2: 1.4 $\mathrm{mm}$, Group 3: $1.4 \mathrm{~mm}$, Group 4: $1.0 \mathrm{~mm}, \mathrm{p}=0.033$ and 0.033 respectively, $90^{\circ}$ : Group 2: $1.5 \mathrm{~mm}$, Group 3: $1.6 \mathrm{~mm}$, Group 4: $1.1 \mathrm{~mm}, \mathrm{p}=0.034$ and 0.009 respectively) and compared to group 1 at $90^{\circ}$ flexion (Group 1: $1.6 \mathrm{~mm}, \mathrm{p}=0.015$ ). 
Tibial First Technique Using Predictive Balancing Improves Joint Balance Compared... Wakelin et al.
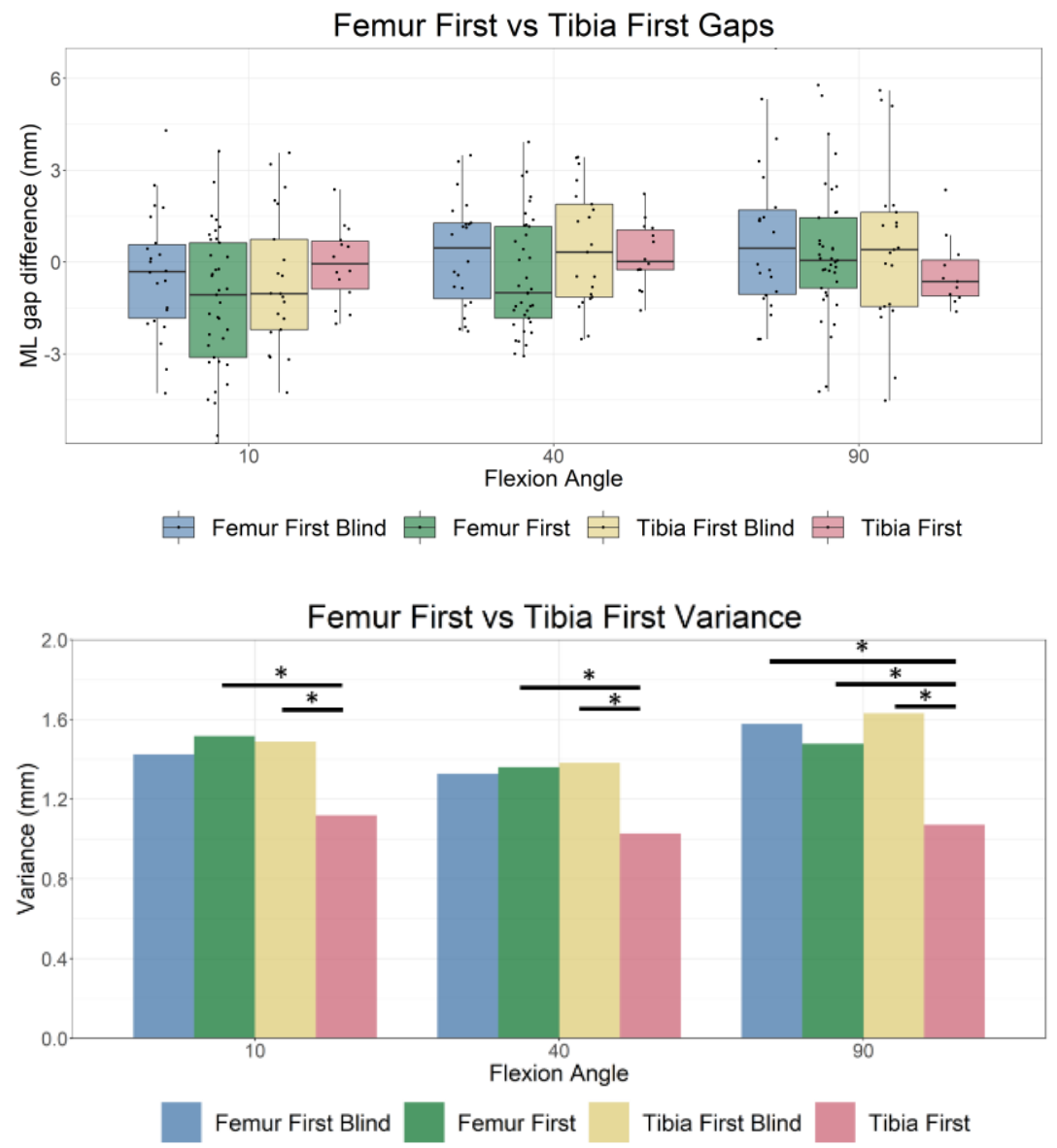

Figure 1 Top: Box plot comparing Medio-lateral gap difference during trialing between the 4 comparison groups. No significant differences were found. Bottom: Comparison of the variance in gap achieved between the 4 groups throughout flexion. The tibia first Non-Blind group achieved the significantly reduced variance across all flexion angles.

\section{Discussion}

The tibia-first technique combined with a digital balancing device significantly reduced the frequency of outliers and gap variance throughout flexion in this prospective consecutive series. No difference in the average ML gap balance indicates an experienced surgeon can achieve a balanced knee across a population, but that the use of a digital balancing tool with a tibia first methodology can improve the precision of gap balancing. The operating surgeon performed femur first measured resection routinely prior to this study. With further experience with the tool and a tibia first method, the variance may further decrease.

The introduction of a digital gap balancing tool using a femur first method as in Group 2 did not change number of outliers, average gap or gap variance compared to Group 1. This highlights the complexity in achieving a balanced knee through soft tissue release and insert thickness after performing the femoral resections using manual instruments. Furthermore, the transition to a tibia first 
technique with manual instruments also did not improve the outcomes measured here, indicating that the combination real time feedback of patient specific gaps under an accurate load prior to the femoral resection is required to reliably balance the knee.

The greatest reduction in variance is at $90^{\circ}$ flexion. The variance in Group 4 does not decrease at $90^{\circ}$, but rather the variance in groups 1-3 increase, potentially reflecting difficulty in isolating joint gap measurements and hip adduction in flexion. Golladay has recently reported improved outcomes in more balanced knees [4] in a measured resection approach, Shelton however, has shown this target may not result in improved outcomes when targeting kinematic alignment [5]. Further work by McEwen has shown improved outcomes with increased lateral laxity in flexion [6]. Ideal joint balance may remain a controversial topic, nonetheless a tibia first approach with digital balancing allows surgeons to accurately achieve a desired joint balance target.

The combination of a tibia first gap balancing technique with a digital gap balancing tool improves a surgeons ability to precisely balance the knee throughout flexion in TKA compared to a femur first measured resection approach.

\section{References}

1. Riviere, C., et al., Alignment options for total knee arthroplasty: A systematic review. Orthop Traumatol Surg Res, 2017. 103(7): p. 1047-1056.

2. Daines, B.K. and D.A. Dennis, Gap balancing vs. measured resection technique in total knee arthroplasty. Clinics in orthopedic surgery, 2014. 6(1): p. 1-8.

3. Shalhoub, S., et al., Development of an Active Soft-Tissue Balancing System for RoboticAssisted Total Knee Arthroplasty, in Handbook of Robotic and Image-Guided Surgery. 2020, Elsevier. p. 459-473.

4. Golladay, G.J., et al., Are Patients More Satisfied with a Balanced TKA? The Journal of Arthroplasty, 2019.

5. Shelton, T.J., S.M. Howell, and M.L. Hull, Is there a force target that predicts early patientreported outcomes after kinematically aligned TKA? Clinical Orthopaedics and Related Research®, 2019. 477(5): p. 1200-1207.

6. McEwen, P., G. Balendra, and K. Doma, Medial and lateral gap laxity differential in computer-assisted kinematic total knee arthroplasty. The bone \& joint journal, 2019. 101(3): p. 331-339. 